\title{
SIGNIFICADO DE LAS PRÁCTICAS TUTORIALES EN LAS ASESORÍAS DE TESIS DE LOS ESTUDIANTES DE MAESTRÍA EN EDUCACIÓN
}

\author{
Meaning of Tutorial Practices in the Counseling Students \\ in Master of Education Thesis
}

\author{
FLOR FANNY SANTA CRUZ TERÁN*1 \\ KONY LUBY DURAN LLARO**2 \\ *Universidad Católica de Trujillo Benedicto XVI. Perú. \\ Correspondencia: santacruz210575@gmail.com \\ **Universidad César Vallejo de Trujillo. Perú. \\ Correspondencia:kony27_teacher@hotmail.com
}

Recibido: 07-09-2017

Revisado: 20-10-2017

Aceptado: 10-01-2018

Resumen: El propósito de este estudio es comprender el significado que atribuyen los estudiantes de maestría en Educación a las prácticas tutoriales durante las sesiones de asesorías de tesis. El trabajo se desarrolló según un enfoque cualitativo con diseño fenomenológico, a partir de la observación participante y la entrevista en profundidad. Los datos se analizaron a través de un conjunto de códigos temáticos, mediante un proceso de codificaciones obtenidas de las unidades de significatividad de un análisis de contenido y de discurso. Los hallazgos permitieron comprender que la comunicación amical, confianza, motivación y buen clima emocional están presentes en las interacciones entre el asesor y el asesorado, lo que muestra la efectividad del recurso didáctico tecnológico en las asesorías.

Palabras clave: fenomenología; tesis; tutoría; posgrado.

\begin{abstract}
The purpose of this study is to understand the meaning that Master of Education students attribute to tutorial practices during thesis counseling sessions. The work was developed according to a qualitative approach with phenomenological design, based on the participant observation and the indepth interview. The data were analyzed through a set of thematic codes, through a process of coding obtained from the units of significance of a content and discourse analysis. The findings allows to understand that friendly communication, trust, motivation and a good emotional climate are present in the interactions between the advisor and the person being counseled, which shows the effectiveness of the technological didactic resource in the counseling.
\end{abstract}

Keywords: phenomenology; thesis; tutoring; postgraduate education.

\footnotetext{
1 Doctora en Administración en Educación de la Universidad Particular César Vallejo (Perú), maestría en Investigación y Docencia Universitaria de la Universidad Católica de Trujillo Benedicto XVI (Perú), magíster en Educación con mención en Docencia y Gestión Educativa. Licenciada en Educación con especialidad en Filosofía, Psicología y Ciencias Sociales. Profesora - investigadora en pre y posgrado de la Universidad Católica de Trujillo y en posgrado de la Universidad César Vallejo.

2 Doctora en Educación y doctora en Gestión Pública y Gobernabilidad; Magister en Educación con mención en Docencia y Gestión Educativa (Universidad Cesar Vallejo, Perú). Licenciada en Educación Secundaria con mención Ciencias Naturales: Biología, Física y Química (Universidad Nacional de Trujillo, Perú). Doctor Honoris Causa otorgado por la Universidad del Golfo (México) y Doctor Honoris Causa por la Universidad Global del Cusco (Perú). Docente de Metodología de Investigación en la Escuela de Posgrado de la Universidad Cesar Vallejo-Trujillo (Perú).
} 


\section{INTRODUCCIÓN}

En los posgrados de investigación no existen cursos, seminarios ni programas especializados para formar a los tutores o asesores de tesis que permitan cumplir con los objetivos académicos de posgrados; por lo que el tutor o asesor sólo se apoya en su experiencia profesional y personal para la realización de la conducción del respectivo asesoramiento del estudiante tutorado o asesorado.

El asesor de tesis cumple una función muy importante para la culminación de los trabajos de investigación de los participantes, por lo tanto es deber de los asesorados cumplir con sus responsabilidades durante la elaboración de la tesis; en esta interacción se pueden presentar ciertas dificultades, por ejemplo la poca disponibilidad en cuanto a tiempo de parte de los estudiantes y del asesor, problemas interpersonales, falta de compromiso por ambas partes. $Y$ también se presenta la situación de que el participante solo aspire a obtener el grado académico por el bono beneficioso que otorga el Ministerio de Educación (2015) en el DS.006-2015-MINEDU, donde se establecen las condiciones y criterios que debe cumplir un docente para obtener el incentivo económico propuesto. También influyen la falta de interés y la poca motivación del estudiante, el cual tendrá que leer exhaustivamente artículos científicos y otras publicaciones para analizar los datos recogidos e interpretarlos, para luego elaborar, desarrollar hasta la culminación de su tesis y presentar un artículo científico para su respectiva sustentación.

Al analizar la problemática presentada se contribuye a la formación de competencias en investigación para desarrollar las capacidades y la obtención de los logros y lineamientos propios de las actividades tutoriales con los planteamientos de los actores. Es por ello que se formula la siguiente pregunta generadora de la investigación: ¿Qué significado le atribuyen los estudiantes de maestría a las prácticas tutoriales en las asesorías de tesis?

Para comprender el significado de las prácticas tutoriales durante las asesorías de tesis se requiere del método fenomenológico, que permite el estudio de los fenómenos subjetivos, y de la hermenéutica que interpreta las interacciones entre los actores durante las asesorías en las prácticas tutoriales, que se manifiesta con sus propias vivencias y experiencias diarias, ya que se carece de información sobre las actividades específicas que se llevan a cabo y desde las cuales los estudiantes viven su experiencia formativa en el proceso de lograr sus competencias investigativas.

Estudios previos señalan que la práctica tutorial debe ser eficiente, acorde a los lineamientos institucionales y la gestión de la tutoría de posgrado (Marinero, 2013), es así que el escenario por excelencia son los procesos de asesorías que se dirigen a ejercitar las habilidades y conocimientos investigativos (Arenas, 2005), siendo el tutor quien requiere de un desempeño humano de alta calidad, creatividad en la visión compartida con el tutorado y con la institución a la cual pertenecen (Vera y Vera, 2013); esta tarea de los tutores no resulta sencilla, no existen instrumentos que guíen su 
desempeño, es por ello que se basan en sus propias creencias y experiencias previas, sin una reflexión continua de su quehacer como formadores (Cruz, Díaz-Barriga y Abreu, 2010). Sin embargo, los asesores se adaptan a las necesidades de los estudiantes para poder apoyarlos, explicar los procedimientos, normas, consecuencias y división de responsabilidades, proporcionando cada vez una mayor retroalimentación (De Kleijn, Meijer, Brekelmans y Pilot, 2014).

Las bases teóricas científicas que sustentan esta investigación son: la teoría del interaccionismo de Blúmer (1982), que tiene como objeto de estudio la interacción, en la cual se atribuye importancia a los significados que las personas expresan de acuerdo a lo que les rodea, y la fenomenología de Husserl (1992), que se centra en descubrir la esencia de la experiencia vivida del ser humano. La teoría sociocultural de Vygotsky (1979) sostiene que el docente asesor actúa como mediador en los aprendizajes del estudiante y es óptimo para el desarrollo de las capacidades y destrezas mediante la sociabilización. La teoría de las representaciones sociales de Moscovici (1986) es la que permite analizar cómo determinado grupo social ve, interpreta y da sentido a una zona de sus vivencias individuales y colectivas, es decir, se orienta a comprender y explicar el pensar del sentido común.

El trabajo de investigación (tesis) cuando se realiza por primera vez siempre presenta dificultades que con el trascurrir del tiempo y la práctica van mejorando, así como con el apoyo y asesoramiento del docente asesor hasta llegar a la redacción final del trabajo. En esta perspectiva, Moreno (2015) está convencida de que investigar se aprende investigando, es por ello la necesidad del desarrollo de habilidades investigativas que se construyen en el investigador según sus actitudes favorables (deseo de saber-hacer y transformar), valores y los conocimientos previos del individuo en formación.

El asesor o tutor de tesis es un docente investigador activo, que tiene la responsabilidad de dirigir la actividad académica del estudiante asociado con el proceso de elaboración de tesis. Por lo cual debe poseer de forma intrínseca valores (responsabilidad, vocación, conocimiento científico, ética, desarrollo intelectual y motivación) de acuerdo al perfil requerido de un buen asesor de tesis (Ramírez, 2001).

Arenas (2005) define al asesorado como el alumno de posgrado en formación, debe manifestar una afinidad creciente con su asesor, con una escucha activa, apertura para el trabajo creativo e independiente, orientación oportuna, pero sobre todo rasgos y actitudes de gran calidad humana. Al respecto, Torres (2013) señala que "el tutorado es un estudiante de posgrado (maestría o doctorado), con claras intenciones de formarse como investigador, incursiona en los estudios de posgrado con un tópico de investigación que sirve de pretexto para iniciar el proceso de formación para la investigación asociada a la relación de tutoría" (p. 5).

Sin embargo, para el presente estudio el asesorado o tutorado es un estudiante de posgrado que realiza un trabajo de investigación y se inicia en el proceso de 
formación para desarrollar competencias investigativas según sus capacidades, destrezas y habilidades que presente.

Las prácticas tutoriales se realizan durante la presentación del borrador del proyecto de investigación, luego el proceso de revisión para el mejoramiento hasta obtener el grado académico. Las interacciones entre los actores (asesor y asesorado) se dan en el plano de una asesoría académica debido a la estructura y objetivos de los programas. De acuerdo a Marinero (2013) la práctica tutorial es un proceso formativo de carácter integral, donde el profesor orienta y monitorea al estudiante para potenciar sus aprendizajes y competencias que le permitan lograr sus metas personales y profesionales.

En este estudio se ha formulado el objetivo general "Comprender el significado que le atribuyen los estudiantes de maestría a las prácticas tutoriales en las asesorías de tesis de una Universidad Privada de Trujillo, con sede Chocope-Perú"; se plantearon los siguientes objetivos específicos: identificar la interacción entre asesor y asesorado que influye en el proceso tutorial, identificar los factores que se relacionan con el desarrollo del investigador en formación, describir las actividades del asesor en las prácticas tutoriales, describir las actividades del estudiante asesorado en las prácticas tutoriales, definir el uso de los recursos didácticos en las prácticas tutoriales para la formación del investigador.

\section{MÉTODO}

El trabajo de estudio se realizó de acuerdo con el paradigma cualitativo con diseño fenomenológico, que busca conocer los significados que cada participante de maestría en educación manifiesta de acuerdo a sus experiencias subjetivas sobre las prácticas tutoriales. Asimismo, se desarrolló de acuerdo al método hermenéutico e inductivo.

\section{Descripción del contexto}

La investigación se realizó en las aulas de una Universidad Privada de Trujillo con sede Chocope, se ubica en la Provincia de Ascope, Región La Libertad-Perú, donde se ofrece el programa de maestría en educación. Esta maestría tiene una duración de cuatro semestres, el curso de desarrollo del proyecto de investigación se dicta en el último semestre (IV) y las clases se dictan cada quince días (sábados y domingos). Se decidió realizar este estudio considerando la accesibilidad del investigador.

\section{Participantes}

Participaron los estudiantes del programa de maestría en educación que trabajan en las Instituciones educativas de Educación Básica Regular (inicial, primaria y secundaria) de dicha provincia, con cargos de formación docente, ocupando algunos 
los puestos directivos en sus respectivos centros laborales que se encuentran ubicados en poblados rurales.

La muestra de estudio estuvo constituida por ocho participantes del último semestre que se encontraban en el proceso de elaboración de su proyecto de investigación. Los participantes tenían un nivel socioeconómico medio alto y sus edades se ubicaron en el rango entre 38 a 65 años. Su participación fue libre y voluntaria.

\section{Instrumentos}

Se utilizó la guía de entrevista en profundidad que permitió obtener la información desde el propio criterio del estudiante, iniciándose con la siguiente pregunta orientadora: ¿Cuál es el significado que le atribuye usted a las prácticas tutoriales durante la asesoría de tesis? Surgiendo luego preguntas abiertas, que permitieron obtener informe a narración de las experiencias vividas del participante, finalizando cada entrevista con la recolección de datos con la saturación teórica. Las entrevistas se realizaron fuera del horario de clases, al culminar sus actividades académicas. Para ello se utilizaron una grabadora de voz y en algunas oportunidades las notas de voz del celular donde quedaron registradas las manifestaciones de cada entrevistado. Tuvieron una duración aproximada de 45 a 50 minutos. Asimismo, para guiar la búsqueda de la información se cumplió con la observación participante realizada durante el proceso de asesoría de las prácticas tutoriales, anotando las evidencias en registros escritos (notas de campo).

\section{Procedimiento}

En el presente estudio de investigación cualitativa se realizó el procesamiento y análisis de los datos de forma manual, lo que permitió realizar una clasificación concreta y personalizada en concordancia a los objetivos formulados. Las técnicas que se utilizaron fueron de acuerdo con el análisis de contenido y de discurso. Luego de recoger los datos de las entrevistas en profundidad y de su transcripción, se realizó una lectura inicial de cada una de ellas lográndose una visión general, y fue necesario leer varias veces las entrevistas para luego realizar una aproximación holística de cada transcripción, sintetizando en frases para interpretarlas posteriormente y cumplir con el análisis acerca del significado del fenómeno que se le atribuye a los participantes. Luego, al transcribir cada entrevista se identificaron, codificaron y categorizaron los principales temas de acuerdo con los datos obtenidos. Es así que se analizó cada transcripción dividiendo el texto en unidades de significatividad, consistentes en una frase con un significado común. Posteriormente, a cada una de estas estructuras se las codificó indicando a qué unidad de significatividad y categoría correspondían, para ello se agrupó la información estableciéndose la relación con la unidad de análisis. Dentro 
de estas categorías se obtuvieron las subcategorías de los mismos testimonios de las entrevistas brindadas por los participantes, con un análisis sistemático por el cual se explicó acerca de la transcripción del texto llegando a una comprensión profunda del significado del fenómeno. Se obtuvo una estructura de cuatro apartados: Código (símbolo de la temática), Estudiante (se colocó la letra inicial de la palabra entrevista, la letra inicial de la respuesta y un número de acuerdo con el orden de la entrevista que se realizó), Testimonio (descripciones significativas) y Significado (interpretación de la investigadora).

\section{RESULTADOS Y DISCUSIÓN}

Tabla 1. Matriz de unidades de significado obtenidos de los datos de la entrevista en profundidad

\begin{tabular}{|c|c|c|}
\hline Unidades de significatividad & Categorías & Código \\
\hline \multirow[t]{4}{*}{ Interacción entre asesor-asesorado } & $\begin{array}{l}\text { - Comunicación en las interacciones } \\
\text { entre asesor y asesorado }\end{array}$ & $\mathrm{ICm}$ \\
\hline & $\begin{array}{l}\text { - Confianza durante las } \\
\text { interacciones }\end{array}$ & $\mathrm{ICn}$ \\
\hline & - Clima en las interacciones & $\mathrm{ICl}$ \\
\hline & $\begin{array}{l}\text { - Motivación durante las } \\
\text { interacciones }\end{array}$ & IM \\
\hline \multirow[t]{2}{*}{ Estrategias didácticas } & - $\quad$ Estrategias de enseñanza & EE \\
\hline & - Estrategias de aprendizaje & EA \\
\hline \multirow[t]{2}{*}{$\begin{array}{l}\text { Acompañamiento en el desarrollo } \\
\text { de las actividades investigativas }\end{array}$} & $\begin{array}{l}\text { - Monitoreo de las actividades } \\
\text { investigativas }\end{array}$ & AM \\
\hline & $\begin{array}{l}\text { - Apoyo en el desarrollo de las } \\
\text { actividades investigativas }\end{array}$ & AA \\
\hline \multirow[t]{2}{*}{ Uso de los recursos didácticos } & - Uso de los recursos físicos & RF \\
\hline & - Uso de los recursos tecnológicos & RT \\
\hline
\end{tabular}

Nota: ICm (Interacciones de Comunicación), ICn (Interacciones de Confianza), ICl (Interacciones de Clima), IM (Interacciones de Motivación), EE (Estrategias de Enseñanza), EA (Estrategias de Aprendizaje), AM (Acompañamiento de Monitoreo), AA (Acompañamiento de Apoyo), RF (Recursos Físicos) y RT (Recursos Tecnológicos). 
Unidad de significado I: Interacción entre asesor-asesorado, la comunicación, la confianza, el clima y la motivación son las categorías percibidas por los participantes.

Mayormente a opinión de los participantes la convivencia durante las prácticas tutoriales ha sido efectiva, producto de una comunicación transparente entre los factores (asesor-asesorado) que permitió un diálogo fluido consolidando las ideas y criterios para el desarrollo del trabajo de investigación.

Durante las interacciones existe una comunicación viable y eficiente en el proceso de convivencia en clase. Hay oportunidad de expresar las ideas y sistematizarlas que servirán para la mejora del trabajo (ER1).

En el asesoramiento de tesis la comunicación es fundamental sobre todo si se realiza de forma frecuente a través de los distintos medios de comunicación. Ramírez (2001) sostiene que el asesoramiento es sinónimo de entrega, desprendimiento, trabajo en equipo, ejemplo y dedicación, apertura y amistad.

La comunicación es buena porque nos viene orientando el trabajo por partes, ya sea en clase, llamadas telefónicas, correo y entrevistas personales (ER5).

Los testimonios anteriores se diferencian de los resultados obtenidos por Arenas (2005), ya que en su estudio se observa la falta de la comunicación entre los actores, es por ello que en sus sugerencias recomienda lo fundamental que es la comunicación para la transmisión de ideas, la corrección del tema y, para superar problemas, que debe ser fluida y motivante, por lo tanto, es un elemento indispensable en una relación interpersonal entre el asesor y el asesorado.

El siguiente participante manifestó que tiene la confianza con el asesor, quien con un manejo ético y profesional ha contribuido a un buen clima durante las asesorías.

Sí existe confianza, porque, se ha establecido el lazo de cordialidad, seguridad y empatía con todo el grupo...mediante un manejo ético y profesional...lo que genera un ambiente armónico y respetuoso (ER8).

Este testimonio hace referencia al manejo ético. Rodríguez (2002) considera que una de las características que debe presentar todo asesor de tesis es la ética, que radica en el profesionalismo que en todo momento debe presentar y la transparencia de su actuación desde el inicio hasta el final de la tesis. Asimismo, afirma que la empatía se encuentra en las interacciones, y es importante en las relaciones interpersonales. El clima en un espacio educativo, llamado aula, nace a partir de las relaciones y vínculos que se establecen entre los que interactúan dentro de ese espacio y que va más allá de lo material ya que se trata de algo más sutil, es decir del clima emocional (Casassus, 2008). Por lo tanto, al establecerse un buen clima emocional se permitió el desarrollo de buenas relaciones interpersonales basándose en el respeto, la honestidad y la sinceridad que favorecen la confianza y el bienestar emocional de las personas. 
En el proceso tutorial el clima emocional es muy importante para que exista una buena relación entre el asesor y el asesorado pero he podido observar que no existe un buen clima emocional... porque algunos asesorados se sienten inseguros de sí mismos y sobre todo en los trabajos realizados (ER7).

La interacción necesariamente es saludable porque existe un buen clima, pero se pudo apreciar que los asesorados al no cumplir con los requerimientos exigidos de su proyecto de investigación generaron la inexistencia de un buen clima emocional, produciéndose la inseguridad y preocupación por el incumplimiento de los objetivos propuestos.

La motivación siempre está presente en el proceso tutorial durante las asesorías: debido a una comunicación permanente, que los incentiva a desarrollar y culminar con su trabajo de investigación, reconociendo su esfuerzo al cumplir finalmente por primera vez una tesis.

La experiencia ha sido grata porque he aclarado mis líneas de conocimiento que ha tenido un límite y con esto me ha permitido buscar más información... me ha ayudado a desarrollar en forma personal y en mi trabajo. Conforme las correcciones que me ha sugerido he ido levantando las observaciones y buscando más información para mejorar mi trabajo. La constante revisión y asesoría me ha ido motivando con sus palabras de aliento a través de los correos que Sí se puede (ER3).

Se puede identificar que la motivación intrínseca está presente junto con la extrínseca. Estos resultados se asemejan al estudio de Cruz, Díaz-Barriga y Abreu (2010) donde indican en una de sus rúbricas el nivel "Ejemplar", que consideran con el más alto calificativo y lo describen así: "Los tutores... motivan, animan, orientan y apoyan a los estudiantes para culminar sus estudios y desarrollar un proyecto de vida" (p. 93).

Unidad de significado II: Las estrategias didácticas, las estrategias de enseñanza y aprendizaje, asesor motivador, asesoramiento.

Se finaliza el seminario de desarrollo del proyecto de investigación con la producción de su trabajo de investigación. Uno de los participantes catalogó como buenas las asesorías y orientaciones que se le brindaron, reconociendo que para desarrollar las capacidades investigaciones se dieron a conocer todos los pasos y procesos en la elaboración del proyecto de investigación y asimismo manifestó que el buen asesor es motivador, brinda confianza y es empático.

En las estrategias de enseñanza, bueno, según... realiza, primero, informa los puntos a tratar, el modo como desarrollarlos y la verificación o revisión de los productos. Es buena porque nos brinda las pautas necesarias en nuestro trabajo, lo que nos ayuda a mejorarlo. De todas las actividades realizadas la más relevante en mi formación como investigadora es el cómo desarrollar el trabajo de investigación, 
siguiendo un proceso riguroso, que permite ir mejorando los conocimientos para el buen manejo de la información.... El buen asesor significa, para mí, mantener motivado al equipo y proporcionarles confianza porque debemos ser empáticos con las personas que son parte de nuestro entorno para demostrar que no tan solo lo académico es parte de la formación sino lo actitudinal (ER1).

Es fundamental que sean considerados como estrategias la explicación, el asesoramiento grupal y personalizado y la exigencia de elaborar buenos o excelentes trabajos de estudio en el proceso del desarrollo de investigación, estratégicamente para el potenciamiento de las capacidades y habilidades investigativas es que se debe conocer la estructura para poder elaborar un trabajo de investigación.

Una buena estrategia que aplica el asesor era citarlos individualmente en la biblioteca para el asesoramiento con la finalidad de que cumplan con toda la estructura planteada en su trabajo de investigación. Un buen asesor es el que brinda confianza, es asertivo y debe tener conocimiento científico para la investigación.

El asesoramiento es correcto con exigencia y depende de cada estudiante su preocupación en culminar bien la investigación. La más relevante para mi formación como investigadora es la investigación teórica del marco conceptual, antecedentes, justificación, objetivos, hipótesis, diseño y la estrategia de citarnos en la biblioteca para investigar. Un buen asesor significa brindar confianza, ser delicado en expresarse, conocer para asesorar y orientar (ER6).

Las estrategias que se desarrollan durante las asesorías son: comunicación directa, orientación, planificación, elaboración y evaluación de acuerdo con lo programado durante cada sesión. Para esta participante las actividades más importantes son el apoyo que le brinda el asesor y una comunicación fluida. Asimismo, considera que un buen asesor es competente, flexible, trabaja en equipo y es orientador.

Las principales actividades durante las interacciones tutoriales es que promueve la comunicación directa y dialoga con sus asesorados, orienta sobre actividades y trabajos relacionados con la tesis, planifica y elabora programa para el desarrollo de trabajo de Tesis y sugiere fuentes bibliográficas y documentales para apoyo de la Tesis. La actividad más importante es la comunicación entre la asesora y el asesorado, seguido de todo el apoyo científico, académico y metodológico que la asesora nos brinda. Se considera un buen asesor, aquel que es competente en el campo del conocimiento elegido, en el tema o trabajo de grado o ser susceptible de interesarse por el tema a tratar, es ser flexible y trabajar en equipo, y aceptar que el estudiante tenga ayuda o asesoría distinta a la suya y también es el que orienta a la mejora y aprendizaje continuo del asesorado (ER8).

Para resolver las inquietudes y dudas que tuvieron los asesorados fue necesario utilizar los medios virtuales como estrategia, teniendo en cuenta el poco tiempo disponible 
para el asesoramiento personalizado y reconociendo la motivación que les permitió el desarrollo y culminación de su trabajo de investigación.

Ha sido importante el trabajo en equipo con la coordinadora de la sede Chocope, permitiendo adecuadamente el desarrollo de su trabajo de investigación. Asimismo, considera que ha logrado desarrollar sus capacidades, habilidades y competencias investigativas.

Las estrategias que se realiza a través de los correos, revisen esto, mejoren. Entraba a los correos y nos decía qué hacer. Cuando yo ya me quería retirar leía los mensajes y decía la profesora se preocupa porque le mandemos nuestros trabajos. Entonces decía, no yo tengo que seguir no me puedo quedar. Con su ayuda y también de los coordinadores, han trabajado en conjunto porque mejoremos y avancemos con nuestro trabajo de investigación y siento que me ido formando en el conocimiento de una investigadora (ER7).

En relación a cómo debe ser un asesor, los participantes manifiestan que debe ser motivador, generar confianza, empático, comprensivo, asertivo, flexible, orientador y tener conocimiento científico. Morales, Rincón y Tona (2005) sostienen que el docente es un investigador modelo, indicando que solo puede llegar a enseñar de forma exitosa si es un investigador, de este modo puede compartir experiencias de forma horizontal.

Se observó que durante las asesorías grupales del proceso de aprendizaje de los estudiantes, compartían información necesaria y se sentían motivados para desarrollar su proyecto. En este proceso se apoyaron mutuamente y a la vez estuvieron agradecidos por la muestra de compañerismo.

Los factores principales que han favorecido a mis aprendizajes durante el proceso de la interacción tutorial es tener la tutoría y orientación durante el desarrollo del trabajo de investigación. También el de compartir con el equipo cierta información que ayudará a mejorar nuestro desempeño en el aula de clases y servirá como aporte para seguir con nuestra meta. Mi participación en las prácticas tutoriales para mi formación como investigadora es en el sentido de la responsabilidad, de investigar más, de ampliar todos estos conocimientos en este largo proceso (ER1).

De acuerdo a lo manifestado por los participantes, utilizaron diversas estrategias de aprendizaje durante el proceso de asesoría en las prácticas tutoriales: ser responsable, buscar información para de esta forma ampliar sus conocimientos, ser perseverante, activo y siempre con el deseo de mejorar, buscando estrategias, como por ejemplo haciendo consultas por los diferentes medios de comunicación (celular, e-mail, WhatsApp), visitando la biblioteca para enriquecer sus conocimientos, compartir información en equipo con sus compañeras, las actitudes y la sociabilidad existente en el grupo de maestría (entre ellas se ayudan y apoyan frente a cualquier dificultad). Moreno (2015) afirma que la identificación de habilidades significativas, así como de 
estrategias son el núcleo fundamental e integrador de los aprendizajes requeridos para hacer investigación de buena calidad.

Sin embargo, afirman haber tenido dificultades durante el desarrollo de sus competencias investigativas y manifiestan lo siguiente:

Las dificultades que tuve fue el haber faltado en un $10 \%$, no haber podido asistir los primeros días por diferentes motivos y lo que más me limita es el factor laboral por el tiempo que me absorbe, soy consciente de rescatar la responsabilidad de cumplir y asistir (ER2).

Los factores que han intervenido, para mí, es un poco frustrante porque sé que puedo dar más, pero factor tiempo es el que me limita para dar al $100 \%$ de todo lo que quiero en mi trabajo de investigación, también la familia, el atender a los hijos. La presión en el trabajo y la exigencia de presentar planes, sesiones y soy directora encargada, docente del $1^{\circ} \mathrm{y}$ al $6^{\circ}$ grado, es absorbente (ER7).

Entre las dificultades que han tenido para considerar una asesoría efectiva y poder cumplir con las capacidades diseñadas del programa de seminario de desarrollo de tesis, es la responsabilidad que tenían como docentes de aula y algunos de ellos por tener asignados cargos directivos. Otras dificultades que limitaban su desarrollo en las competencias investigativas era el factor familiar (atención a familia) y el factor tiempo.

Unidad de significado III: El acompañamiento en el desarrollo de las actividades investigativas, monitoreo y apoyo.

En todo proceso de investigación el desarrollo del aprendizaje está basado en la decisión por investigar llevándola a la práctica y requiriendo de la orientación de una persona experta en la investigación científica.

En cuanto al proceso de asesoría no me puedo quejar, de muestra está los correos que usted me enviaba cuando yo no hecho presente mi trabajo y me hacía saber que tenía que enviar mi trabajo corregido. Están los correos donde usted me estimulaba. Ahí me brindaba los asesoramientos y me hacía recordar: no se olvide profesora el trabajo, tal fecha hay que presentarlo y a veces yo con un poquito de demora, haciéndolo llegar a su correo le enviaba y por la mañana, por la tarde o por la noche o al día siguiente yo ya tenía respuesta, ya me llegaba su respuesta de que estaba bien o estaba mal y en que tenía que mejorar o hacía falta. En cuanto al asesoramiento, estoy muy satisfecha, porque cada vez que le he solicitado, usted me ha respondida cada consulta que he solicitado (ER6).

El monitoreo y evaluación son los que brindan información para ser tomada en cuenta en la mejora de las actividades a realizar, siendo necesario reorientar el ritmo de trabajo con una nueva planificación (Berumen, 2010). 
El proyecto de investigación, considerando las asesorías, se desarrolló en el tiempo programado de acuerdo al cronograma establecido por el programa de maestría en educación, afirmando los participantes en su mayoría que cumplieron con el tiempo estipulado.

El tiempo que se desarrolla para la asesoría es el adecuado, porque aparte de los días de clase la asesora me brinda orientaciones en otros horarios (RE4).

En la categoría de Apoyo (AA) y según las subcategorías se analizó cada uno de sus testimonios, en los cuales manifiestan que el apoyo siempre está presente, es permanente haciendo las observaciones y correcciones a su trabajo de investigación.

El apoyo que brinda la asesora en mi formación como investigadora, se resume en lo siguiente: Me ayudó a encontrar y desarrollar habilidades y conocimientos en un campo específico del saber, específicamente habilidades para la investigación que me permitan realizar la investigación. Me ayudo a potenciar mis destrezas en un campo específico para así entender los problemas del ámbito académico y laboral (ER8).

El proceso formativo es de carácter integral en la práctica tutorial, basado en la orientación y seguimiento por parte del profesor, encaminado a potenciar el desarrollo de aprendizajes significativos en el estudiante y, con ello, las competencias necesarias para permitirle el logro de sus metas personales y profesionales (Marinero, 2013).

Por lo cual el apoyo brindado es una contribución a su formación en el desarrollo de competencias para la investigación, porque se les brindan orientaciones, explicaciones y revisiones permanentes durante el proceso de desarrollo del proyecto de tesis. El acompañamiento, apoyo y monitoreo es incondicional, constante, siempre con la predisposición para asesorar. Así como también estuvo dirigido a potenciar sus destrezas con la búsqueda de información utilizando libros en físico (Biblioteca UCV), libros virtuales (googlebooks), brindando herramientas virtuales para la búsqueda de antecedentes internacionales (google académico), al igual que el análisis e interpretación de los datos obtenidos.

Estos resultados se pueden contrastar con el estudio realizado por Vera y Vera (2015) donde los encuestados afirman que existe un alto desempeño de acompañamiento de parte de los docentes, así como de sus destrezas cognitivas, condiciones personales y funciones como tutor siendo un influente en la alta productividad científica, vinculado a la elaboración y presentación de la tesis. Asimismo, De Kleijn, Mainhard, Meijer, Brekelmans y Pilot (2014) sostienen que los estudiantes perciben la retroalimentación y autorregulación de tareas mediante los asesoramientos, comprendiendo qué pasos van a seguir, por lo cual perciben que están aprendiendo más de su supervisor de tesis (asesor) encontrándose satisfechos. 
Unidad de significado IV: Del uso de los recursos didácticos en la categoría de recursos didácticos físicos (RF) y de recursos didácticos tecnológicos (RT).

Los recursos didácticos son los medios y materiales que auxilian la labor del formador al simplificar la comprensión de los contenidos (Instituto Nacional para la Educación de los Adultos, s.f.). Por lo cual van a ser útiles durante el proceso académico, propiciando la atención y el aprendizaje.

El uso de los recursos didácticos físicos que se encuentran presentes en los procesos tutoriales, según los testimonios de los participantes:

Los recursos físicos que intervienen en las prácticas tutoriales para mi formación como investigadora es el manejo de información (módulos del curso), proyección de diapositivas, bibliografía (libros), entre otros (ER1).

Con carpetas, proyectores, una laptop y una mini biblioteca que la coordinadora lo adecuado para poder tener información a través de libros (ER3).

El recurso didáctico tecnológico utilizado durante las prácticas tutoriales es Internet, esto facilitó la comunicación e interacción entre el asesor, asesorado y los temas de estudio, acercando a los participantes a la realidad del tema estudiado.

Y en cuanto a lo tecnológico, aquí...si tenemos el internet, pero sería importante que todas tuviéramos máquina (o sea una computadora o laptop) para ir avanzando y desarrollando nuestro trabajo aquí mismo, con sus orientaciones. Sería más enriquecedor (ER7).

Identifican la necesidad de tener una computadora o laptop para que puedan trabajar en grupo al mismo tiempo y ritmo; lo que permitió avanzar el trabajo de investigación en el tiempo adecuado, como también les sirvió para mejorar su aprendizaje en investigación científica.

Aguirre y Ruíz (2015) manifiestan que en estos tiempos el docente en las asesorías no solo desarrolla competencias de la metodología sino también lo pedagógico, didáctico y digital. Afirman que es importante que todo docente que enseña investigación (asesor de tesis) se apropie de las tecnologías para el diseño de ambientes de aprendizaje colaborativos que faciliten el acompañamiento pedagógico de quien elabora una investigación en la modalidad de tesis.

Finalmente, es conveniente manifestar las limitaciones que se han presentado en el presente trabajo de investigación cualitativo. No se encontraron trabajos previos relacionados al tema de estudio a nivel local y nacional, pero a nivel internacional fueron muy pocos los que se relacionaron de forma directa con estos temas, siendo esto una dificultad para hacer el análisis de contraste de similitudes o diferencias de acuerdo con los resultados analizados. Sime (2015) afirma que existe una limitada diversidad y fundamentación de metodologías de estudios cualitativos sobre posgrado 
en América Latina, así como también presenta una compilación de estudios de los procesos de asesoría de tesis en posgrado en educación, encontrando tan solo 6 artículos internacionales seleccionados que fueron publicados entre el 2007 y el 2013 (Sime y Revilla, 2014).

\section{CONCLUSIONES}

En el contexto de la interacción entre asesor y el asesorado durante las prácticas tutoriales, la comunicación se presentó de forma amical, directa, viable y sincera entre los actores y en la medida que transcurrió esta relación se logró la confianza necesaria para formular las preguntas en forma sincera, despejando las dudas sobre los hechos que requieran una aclaración. De la misma forma se refirió al ambiente de las interacciones como ameno, tranquilo, bueno y agradable lográndose un buen clima emocional. Se encontró la empatía en las relaciones interpersonales. También se puso de manifiesto lo difícil que es para algunos participantes una buena comunicación, lo que impidió que existiera la confianza necesaria en la interacción asesor-asesorado, lo que probablemente podría presentarse como una desmotivación porque no se expresó lo que se sentía o lo que se pensaba, inhibiendo sus emociones. Esto se puede traslucir por la inseguridad que sentían por las inasistencias a las asesorías o tardanzas, originándose una preocupación y frustración en el logro de las competencias investigativas para la elaboración de su tesis.

La aplicación de estrategias fue lo más adecuado en el proceso de enseñanzaaprendizaje durante las asesorías en las prácticas tutoriales. Las orientaciones que se les brindó permitieron la formación para el logro de las competencias investigativas, a saber: la revisión, explicación, asesoramiento personalizado, comunicación, planificación, elaboración y evaluación de acuerdo a lo programado. Por ello es que el docente en asesoría de investigación en posgrado debe estar interesado en conocer a sus estudiantes y aplicar las estrategias motivadoras para un mejor aprendizaje.

Los factores que se relacionan con el logro de las competencias investigativas del estudiante de maestría son el factor académico y emocional, que influyeron como factores positivos, interviniendo las estrategias didácticas de enseñanza y cuyos procedimientos de estrategias utilizados son indispensables para desarrollar sus capacidades investigativas. Asimismo, durante el proceso de interacción surgieron las estrategias didácticas de aprendizaje, reconociendo la exigencia y esfuerzo que implica realizar un trabajo de investigación en maestría. Sin embargo, el no cumplir en el tiempo indicado con la presentación de los trabajos de proyecto de investigación, el faltar a las sesiones de asesorías programadas y no cumplir con las indicaciones fueron acciones justificadas por la recarga laboral, responsabilidad familiar y el tiempo que disponen, incidiendo de forma negativa en el logro de las competencias investigativas. 
La actividad que realizó el asesor en las asesorías durante las prácticas tutoriales en la formación para la investigación de los estudiantes fue el acompañamiento en el desarrollo de las actividades investigativas; teniendo como categorías el monitoreo y la evaluación. Para el asesoramiento personalizado el docente (asesor de tesis) distribuyó su tiempo para cada estudiante. El apoyo metodológico que se brindó fue permanente, incondicional, constante a través de las orientaciones, explicaciones y revisiones permanentes al trabajo de investigación. La asesoría a través de los diferentes medios de comunicación (telefónicos e informáticos) fue una estrategia de apoyo importante que contribuyó a su desarrollo en las habilidades investigativas y de las actividades de participación del asesorado para su formación en el logro de sus competencias investigativas, siendo éstas: ser responsable, perseverante, activo y buscar estrategias para el desarrollo de habilidades y destrezas para el logro del conocimiento científico.

Los recursos didácticos presentes durante las prácticas tutoriales utilizados fueron los físicos: módulos del seminario de elaboración y desarrollo del proyecto de tesis, diapositivas, proyector, separatas, material de escritorio, pizarra, libros y revistas. Asimismo, sobre los recursos didácticos tecnológicos sostuvieron que Internet y los medios de comunicación (el celular, el correo electrónico, WhatsApp) han sido medios que permitieron las constantes comunicaciones para las oportunas orientaciones del asesor.

Finalmente, este estudio con método fenomenológico-hermenéutico permitió comprender las experiencias vividas por los estudiantes, con quienes se pudo establecer una empatía y que sus vivencias y experiencias obtenidas durante las asesorías de tesis en las prácticas tutoriales sirvieron para lograr sus competencias investigativas en la elaboración de sus tesis.

\section{REFERENCIAS}

Aguirre, G., y Ruíz, R. (2015). TIC en el apoyo a la enseñanza en la investigación y las asesorías de tesis. Ventana Informática, 32, 11-27. Recuperado de https://goo.gl/MRqfze

Arenas, J. (2005). El proceso de asesoría en dos programas de maestría del área de educación y humanidades de la Universidad de Colima (Tesis de maestría). Universidad de Colima, Colima, México. Recuperado de http://digeset.ucol.mx/tesis_posgrado/Pdf/Jose_Juan_Arenas_Velasco.PDF Berumen, J. (2010). Monitoreo y evaluación de proyectos. Medellín, Colombia: Escuela Latinoamericana de Cooperación y Desarrollo.

Blúmer, H. (1982). El interaccionismo simbólico: perspectiva y método. Barcelona, España: Hora D.L. Casassus, T. (2003). La escuela y la (des)igualdad. Santiago, Chile: LOM

Cruz, G., Díaz-Barriga, F., y Abreu, L. (2010). La labor tutorial en los estudios de posgrado. Rúbricas para guiar su desempeño y evaluación. Perfiles Educativos,23(130), 83-102. 
De Kleijn, R. Mainhard, T., Meijer, P., Brekelmans, M., y Pilot, A. (2013). Master's thesis supervision: relations between perceptions of the supervisor-student relationship, final grade, perceived supervisor contribution to learning and student satisfaction. Studies in Higher Education, 37(8), 925939. https://doi.org/10.1080/03075079.2011.556717

De Kleijn, R., Meijer, P., Brekelmans, M. y Pilot, A. (2014). Adaptive research supervision: exploring expert thesis supervisors' practical knowledge. Higher Education Research \& Development, 34(1), 117-130. https://doi.org/10.1080/07294360.2014.934331

Husserl, E. (1992). Invitación a la fenomenología. Barcelona. España: Paidós

Instituto Nacional para la Educación de los Adultos. (s. f.). El formador de asesores. Recuperado de www.conevyt.org.mx/colaboracion/servicios/formadorasesores.pdf

Marinero, M. (2013). La gestión de la tutoría a nivel de posgrado. Diagnóstico de la práctica tutorial del 2009 al 2012, en una entidad académica en una universidad Veracruzana (Tesis de Maestría). Universidad Veracruzana, veracruz, México. Recuperado de http://cdigital.uv.mx/bitstream/123456789/34810/1/marinerouribe.pdf

Ministerio de Educación. (2015). Decreto Supremo № 006-2015-MINEDU. Recuperado de https://goo.gl/gh5ASq

Morales, D., Rincón, A., y Tona, J. (2005). Como enseñar a investigar en la universidad. EDUCERE, 9(29), 217-224.

Moreno, M. (2015). La formación para la investigación en posgrado: Una propuesta de desarrollo de habilidades. En V. Mancovsky (Coord.), La formación para la investigación en posgrado (pp. 28111). Buenos Aires, Argentina: Centro de Publicaciones Educativas y Material Didáctico.

Moscovici, S. (1986). Psicología social. Barcelona, España: Paidós.

Ramírez, C. (2001). Modelo integral para llevar a cabo una tesis en Ciencias Sociales. México D.F., México: Limusa.

Rodríguez, M. (2002). Hacia una nueva orientación universitaria: modelos integrados de acción tutorial, orientación curricular y construcción del proyecto profesional. Barcelona, España: Universidad de Barcelona.

Sime, L., y Revilla, D. (2014). Aportes a los procesos de asesoría en posgrados en educación. Lima, Perú: Escuela de posgrado de PUCP.

Sime, L. (2015). Los estudios cualitativos sobre los posgrados en educación en América Latina. Recuperado de http://proceedings.ciaiq.org/index.php/ciaiq2015/article/view/262/258

Torres, J. (2013). En busca de habitus científicos: La tutoría en un doctorado en educación. Recuperado de https://goo.gl/6sDoo3

Vera, L., y Vera, A. (2015). Desempeño del tutor en el proceso de acompañamiento en la producción científica. Revista de Estudios Internacionales en Ciencias Sociales. 17(1), 58 -74. Recuperado de http://publicaciones.urbe.edu/index.php/telos/article/viewArticle/3832

Vygotsky, L. (1979). El desarrollo de los procesos psicológicos superiores. Buenos Aires, Argentina: Grijalbo. 